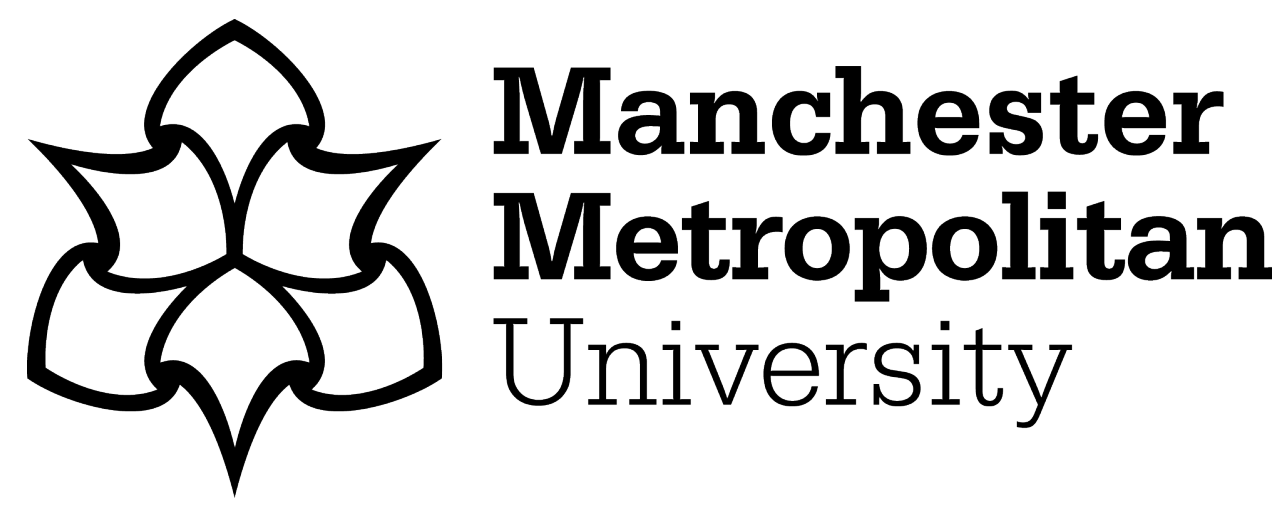

Hearn, Jasmine Heath, Finlay, Katherine Anne and Fine, Philip A (2016) The devil in the corner: a mixed-methods study of metaphor use by those with spinal cord injury-specific neuropathic pain. British Journal of Health Psychology, 21 (4). pp. 973-988. ISSN 2044-8287

Downloaded from: https://e-space.mmu.ac.uk/622470/

Version: Accepted Version

Publisher: Wiley

DOI: https://doi.org/10.1111/bjhp.12211

Please cite the published version 


\title{
The devil in the corner: A mixed-methods study of metaphor use by those with spinal cord injury-specific neuropathic pain
}

\section{Keywords: qualitative, IPA, SCI, chronic pain, imagery, content analysis}

\begin{abstract}
Objectives. Metaphorical expressions of persistent pain play an influential role in the modulation of pain. This may be particularly distressing for those with physical disabilities such as spinal cord injury (SCI). Neuropathic pain (NP) after SCI is often described using metaphorical expressions such as burning and electricity. This study explored the use of metaphors by those with NP after SCI.
\end{abstract}

Design. A qualitative, semi-structured interview design was employed.

Methods. Data were analysed using Content Analysis (CA) and Interpretative Phenomenological Analysis (IPA) to explore prevalence of metaphor use, and its meaning. Sixteen individuals aged between 23 and 82 years, with chronic NP (persisting for three months or longer), arising from SCI were interviewed in their homes, or on hospital wards $(\mathrm{M}=10, \mathrm{~F}=6)$. Interviews lasted between 40 and 120 minutes.

Results. The results capture a range of metaphorical expressions embedded in participants' accounts. Three themes emerged; (1) Pain as a Personal Attack; (2) The Desire to be Understood; and (3) Conveying Distress Without Adequate Terminology. CA revealed that younger age, female gender, and outpatient status were associated with increased metaphor use.

Conclusions. This study highlights the power of metaphor in eliciting understanding of NP after SCI from others, whilst demonstrating the challenge of communicating NP. Cognitive treatment that incorporates image-based techniques with acceptance and mindfulness-based therapies may encourage adaptive responses to, and interpretation of, pain. This may subsequently reduce pain-related distress and catastrophising. 


\section{Introduction}

Over $60 \%$ of people with spinal cord injury (SCI) live with chronic pain (Kennedy, Frankel, Gardner, \& Nuseibeh, 1997; Modirian, Pirouzi, Soroush, Karbalaei-Esmaeili, Shojaei, \& Zamani, 2010). Such pain is often reported as severe or excruciating, and is likely to persist over the first five years after injury (Siddall, McClelland, Rutowski, \& Cousins, 2003). Neuropathic pain (NP) arises as a result of damage to nerves in the spinal cord (Werhagen, Budh, Hultling, \& Molander, 2004), and is often described using burning, electric, and crushing descriptors, and can be intrusive and distressing (Bennett, 2001).

Metaphors associated with pain are examples of negative cognitions, reflecting perceptions and emotions. Exposure to such cognitions is associated with increased distress and pain intensity in chronic pain populations (Philips, 2011). For those with physical limitations such as SCI, metaphor use may contribute towards the development and maintenance of psychological disorders (Holmes, Arntz, \& Smucker, 2007). Such negative impact suggests that language use by those with chronic NP may be an important moderator of pain experience. Despite such evidence, however, metaphor use by those with physical restrictions, has received little attention, as it is not often the direct focus of analyses (Smith $\&$ Sparkes, 2004). Awareness of the impact of metaphors in language, therefore, will improve both understanding, and the ability of healthcare professionals (HCPs) to identify those most at risk of distress, and offer relevant support.

Metaphors can be used as a form of identity construction for those whose lives are disrupted by illness (Becker, 1997). However, over time, the commonly used vocabulary for pain expression is becoming increasingly restricted by the development of pain measurement tools (Bourke, 2014) such as the McGill Pain Questionnaire (Melzack, 1975), and as such, metaphors encourage elaboration on sensorimotor qualities that others may not personally understand (Radley \& Chamberlain, 2001). Consequently, shared understandings from others 
may be invoked, thus enabling them to provide enhanced support (Howe, 2008), and as such, metaphors may be highly suited to the expression of NP after SCI.

The current research evolved from previous work exploring chronic NP after SCI. The original studies (BLINDED, UK, 2015) analysed semi-structured interviews using Interpretative Phenomenological Analysis (IPA; Smith, Flowers, \& Larkin, 2009) to explore the chronic NP experience in its own right, focusing upon the similarities and differences between inpatients and outpatients. Strikingly, upon revisiting the data, 15 of the 16 participants used metaphorical expressions when describing pain experiences, suggesting that they were engaging in meaning-making; attempting to make sense of NP. Enriched understanding, therefore, may be reached by returning to the original transcripts with a focus on rates of metaphor use, and functions of such language. This can provide a foundation for evidence-based integration of imagery-based interventions with cognitive-behavioural techniques (Jamani \& Clyde, 2008).

\section{Methods}

\section{Participants}

Ethical approval was obtained from local R\&D, National Research Ethics Committee (reference no: $\mathrm{XXX}$ ), The $\mathrm{XXX}$, and The University of $\mathrm{XXX}$ (trial registration no: $\mathrm{XXX}$ ). Participants were self-selecting, in a purposeful sample, such that they had experiential knowledge of NP (Smith \& Osborn, 2003). Inclusion criteria included: SCI for at least three months; NP present for a minimum period of three months, in order to meet the International Association for the Study of Pain (2011) definition of chronic pain; no significant cognitive impairment; 18 years or over; English speaking, due to the nature of the qualitative methodology. 
The final sample $(N=16, \mathrm{~F}=6, \mathrm{M}=10)$ consisted of eight outpatients and eight inpatients, all of whom had been diagnosed with chronic neuropathic pain and SCI. The gender divide reflects population norms indicating higher incidence of SCI in males. Ages ranged from 23 to 82 years old $(M=53.2 S D=19.5)$. Average pain intensity ranged from three to ten $(M=6.43, S D=2.89)$, as rated on a visual analogue scale. Level of injury ranged from C2 to L2 (levels of spinal nerves, with $\mathrm{C} 1$ being the highest, located in the cervical spine, and S5 being the lowest, located in the sacrum; higher levels of injury are associated with increased physical impairment). Participant demographics are presented in Table 1. Pseudonyms are provided to preserve confidentiality and anonymity.

\section{***Insert Table 1. Participant demographics. ***}

\section{Procedure}

Hospital staff approached eligible patients with study information. Those interested were introduced to the researcher, provided with further, detailed information, and offered an opportunity to ask questions. Participants were given two weeks to consider their consent, after which, times, dates, and locations of interviews were arranged. Written, informed consent was obtained prior to interviews, which were conducted in participants' homes $(\mathrm{N}=$ 7) and in private rooms in hospital $(\mathrm{N}=9)$. Interviews were conducted by the first author, lasting between 40 and 120 minutes. Interviews were audio-recorded on an encrypted device, and transcribed verbatim, prior to analysis.

\section{Data Collection}

Data collection followed the systematic approach of IPA, as described by Smith, Flowers, and Larkin (2009). Data were collected by an open-ended, semi-structured interview 
schedule (see Table 2). Each interview began with demographic questions, followed by a broad opening question, 'Tell me about your experience of pain since your SCI'. Participants were free to lead the interview, unrestricted by the imposition of topics, to enable discussion of their experience in terms of their own personal context. The interview schedule was designed to be as comprehensive as possible, and the interviewer referred to it to ask questions only where areas had not been covered by the participant.

\footnotetext{
***Insert Table 2. Interview Schedule.***
}

\section{Analysis}

Transcribed interviews were read numerous times prior to conducting IPA, as recommended by Smith, Flowers \& Larkin (2009). Todd and Harrison (2008) suggest that if the aim of research is not purely linguistic (and given that pain is experienced socially, as well as physically), thematic induction may be useful for metaphor analysis. This involves examining and recording themes within data to be able to describe the phenomenon. Considering that the overall framework of the original (IPA-based) study is interpretative, rather than linguistic, returning to the data required two stages. First, Content Analysis (CA; Berelson, 1952) was adopted to provide the frequencies of metaphor use, and consisted of rereading each transcript, highlighting metaphors used when describing pain. The second author validated the analyses, identifying metaphors that may not have been noted originally. Summative scores were calculated for individual participants, and multiple occurrences of the same metaphors were counted individually.

The second stage utilised IPA in such a way that focused specifically upon interpretation of metaphors. Each transcript was re-read whilst linguistic, descriptive, and conceptual comments and notes were recorded. Metaphors for each participant were clustered 
according to conceptual similarities. When all accounts were analysed, a cross-case analysis was conducted and a master table created. The researcher analysed iteratively, moving between part and whole, revisiting data to ensure that interpretations remained grounded, as recommended by Smith, Flowers, \& Larkin (2009).

A reflective diary was used in determined efforts to 'bracket off' prejudgements, so as to remain true to each individual's account (though it is acknowledged that the epistemological stance of the researchers may have played a role in the interpretations). Interpretations presented in this paper are a result of the researchers' interpretations of participant accounts, and are considered credible and meaningful, as a result of the use of two independent auditors, who have experience with chronic pain patients, or IPA, following recommendations by Smith and colleagues (2009). Auditors checked super-ordinate themes and corresponding quotations, discussing thoughts and interpretations, thus illuminating understandings of themes further and ensuring themes were grounded in the data. Any disagreements were resolved through discussion and re-working of themes. Themes were reduced and prioritised in terms of prevalence, richness of metaphors, and their ability to highlight the nature of the themes, and experience of NP. The results also represent an analysis based upon open discussions surrounding pain, as opposed to pain-language use specifically.

\section{Results}

\section{Content Analysis}

Of the 16 participants, 15 used metaphors when describing pain. Levene's test indicated equal variances $(F=2.18, p=.002)$ showing that variance between inpatients and outpatients differed significantly. A chi-square test of independence was conducted to explore 
the relationship between patient status (in- vs. outpatient) and metaphor use. Outpatients used significantly more $\left.\mathrm{X}^{2}(2, N=16)=14.92, p=.001, \mathrm{~V}=.36\right)$, and wider range $(p=.003, \mathrm{~V}=$ .32) of metaphors than inpatients.

The total number of metaphors used by all participants was 115 . The most commonly used metaphors were heat-related, followed by 'pins and needles', and 'electricity'. Outpatients most commonly used thermal descriptors, whilst inpatients most frequently used 'pins and needles'. The total, and range, of metaphors used by each participant are in Table 3.

\section{***Insert Table 3. Participant use of metaphors. ***}

Mann-Whitney $U$-tests were conducted, comparing metaphor use in terms of gender, pain intensity, time since injury, and age, for exploratory purposes. These are presented in Table 4. Females used metaphors significantly more often than males $(U=886 p=.001, r=$ -.40), whilst those aged 50 or over used significantly fewer metaphors than those aged 49 and under $(U=132.5, p=.001, r=-.71)$. No other significant results occurred. Initial analyses of time since injury were conducted on data that divided the group into two categories (up to 12 months post-injury, and more than 12 months post-injury). These data indicated nonsignificant results. However, upon returning to the data and dividing participants between up to 24 months post-injury and more than 24 months post-injury led to significant differences, indicating that those who were within two years of injury were significantly more likely to use metaphors than those who were over two years post-injury $(U=957, p=.007, r=.25)$.

***Insert Table 4. Results of Mann-Whitney U-tests exploring metaphor use.***

\section{Interpretative Phenomenological Analysis}


Three themes were identified using IPA: (1) Pain as a Personal Attack, (2) The Desire to be Understood, and (3) Conveying Distress Without Adequate Terminology. These themes detailed participants' engagement with their experiences of pain.

Pain as a Personal Attack. Participants discussed psychological anguish surrounding the uncontrollability of pain, likening it to an embodied entity relentlessly attacking them. Seven of the sixteen, (outpatients $\mathrm{N}=5$, inpatients $\mathrm{N}=2$ ), described an attacker, and their futile fight against it. Daniel considered himself a victim of pain:

$[\text { The }]^{1}$ pain will just be like [. . $]^{2}$ it's just, just like someone's hitting you.

Daniel, outpatient: line 147

It's like some little devil in the corner. Yeah, you know like that little exorcist thing in the corner ... ${ }^{3}$ You just think of a bad thing ... why is someone torturing me?

Daniel, outpatient: lines 358-360

Daniel describes his pain as a devil, inducing a powerful image, suggesting that there exists a struggle against a malevolent, uncontrollable, embodied pain. Such language highlights psychological anguish and a perception of pain as a torturous punishment, to which he is victim, inducing psychological distress, fear, and further physical pain. This may be further exacerbated by his more recently sustained injury (two years prior to interview), and may suggest that he is yet to find ways of coping with this relatively new experience.

James, also two years post-injury, adds further support to this theme of embodied others:

\footnotetext{
${ }^{1}$ Descriptive information added by the author

${ }^{2}$ Indicates pause of two seconds

${ }^{3}$ Indicates where text has been removed in order to improve coherence of quotations
} 
... you're doing whatever you're doing ... and a little person inside flicks a switch and shuts you down ... literally just collapse ... that's the only way I can describe it, it is, it's horrible.

James, outpatient: page 14, lines 330-332

James describes his pain as something that is out of his own control, unable to do anything but collapse under the pain. James' idea of pain as out of his control suggests that he has an external locus with regard to pain and pain management, and that he is a passive victim of pain, illustrating his feelings of helplessness. The metaphor used emphasises pain's uncontrollability.

Jimmy also felt a passive victim:

I do wonder if this is my purgatory sometimes, the pain.

Jimmy, inpatient: line 179

Jimmy, one year post-injury, relates his pain to being trapped in purgatory, as though NP is a form of punishment for personal sins. His quote illustrates the damaging nature of pain upon his psychological well-being, as well as a catastrophic perception of himself as a passive victim of pain that is out of his own control.

In contrast, Rebecca is 21 years post-injury, and described pain as uncontrollable with a matter-of-fact approach, and considerably less distress:

It seems to be, it quietens down for a bit, and then it sort of rears its ugly head, and then gets, can be severe, really bad, not so bad, whatever.

Rebecca, outpatient: lines 118-119

The phrase 'rears its ugly head' conjures the image of pain appearing as an undesired entity unpredictably in Rebecca's life. The metaphor within this sentence offers a layer of emotion that the listener may be better able to relate to, drawing attention to the idea that Rebecca thinks of pain as 'ugly' and unwelcome, but that she feels better able to cope with it.

The threat of pain, and the participants' perceived vulnerability is highlighted within this theme, through their use of language that illustrates the emotional impact of NP. Feelings 
of helplessness appeared common, with time since injury potentially playing a role in the appraisal of NP.

The Desire to be Understood. Participants described NP in terms of painful stimuli that might have been previously experienced by the listener, thus their choice of metaphors became windows to the experience, allowing for a deeper understanding to be reached. Following initial descriptions, they then graduated these to emphasise the intensity and distress experienced in order for the listener to imagine something similar to the initial descriptions provided. Interestingly, those voicing this theme most strongly, and thus presented below, were within two years of injury, again suggesting that time since injury may play a role in the appraisal of NP, and providing depth to the content analysis.

Pins and needles were a commonly used metaphor:

... it's all like pins and needles and, uh, a bit stronger than pins and needles, it almost feels like nails.

Deb, inpatient: lines 5-6

Well it's like pins needles but like a more, harder, do you know what I mean? A bit more intense, more intensive pins and needles. And then you get like an odd thunder strike, as if lightning's taken your leg.

George, inpatient: lines 3-4

Both Deb and George use pins and needles, but identify that NP is much worse than this, advancing their accounts to 'intense' and 'stronger'. Starting with descriptions that may be understood by the listener before graduating these allowed for the listener to begin to empathise. The escalations to 'thunder strikes', 'lightning', and 'nails' then allow the listener to imagine the intensity, and thus an improved understanding may be reached.

Another phenomenon that those without SCI may have experienced is toothache: 
... it's like having a continuous toothache that just will not go away... But it's worse pain than a toothache.

Jennifer, inpatient: lines 73-74

Jennifer's comparison of NP is to a toothache, something that those without SCI may be able to understand. Jennifer also graduated her description, telling the listener that NP is worse than the original description, yet struggling to provide words to illustrate this.

This illustrates the desire that those with NP have to be understood by those with whom they engage in social situations. This desire led participants to actively search for and use comparisons in order to facilitate such understanding, despite not having adequate words to explain the actuality of NP.

Conveying Distress Without Adequate Terminology. All participants voiced distress associated with NP, and 15 of 16 resorted to the use of metaphors for this task. They often listed multiple metaphors due to its unique and ever-changing temporality and its impact upon their wellbeing, suggesting that they felt no adequate adjective exists for the description of the experience of NP in its entirety. Again, those presented within this theme were within two years of injury.

Some participants chose to describe their pain using thermal descriptors. Emma found NP particularly distressing, and used metaphors in terms of heat:

What I meant by sitting in a pit of fire ... you've got every nerve ending that's just going hellfire, and you just don't know what to do with yourself.

Emma, outpatient: lines 189-191

Emma's quote encapsulates the distress voiced by many participants, and the sheer intensity with which her pain is experienced. Her metaphor demonstrates how NP feels, and the sheer intensity of it, almost like an inescapable torment, whilst also demonstrating its impact on her ability to manage her pain. However, this is not a description that can be understood by the listener, and may represent attempts to convey the distress induced by NP. 
Alice presented a fusion of the experience of embodied pain and thermal descriptors: I get fuzzing, burning, tingling ... it stings ... it changes all the time, it's never the same. Feels like something's crawling inside you, horrible.

Alice, inpatient: lines 14-17

The listing of pain-related adjectives and metaphors illustrates Alice's struggle to find the most suitable descriptor, as well as the distress induced by the unpredictable, everchanging nature of her pain. Alice says herself that her experience is horrible, utilising metaphors that may invoke disgust responses, despite the fact that this is not necessarily something that the listener can relate to.

Daniel also contributed to the theme:

You're just like [. .] it's it's crazy, it's like someone just [. .] it's like sometimes someone could just brush my leg and it'll just go bang, and just, it's like someone's just given you an electric shock [. .] and it feels like, sometimes afterwards my leg is burning. Feels like literally someone's just poured a kettle on my leg.

Daniel, outpatient: lines 109-112

Daniel graduates his description from electric shock towards more extreme imagery of boiling water. This illustrates the sudden onset of pain, and instances of shock when it occurs, whilst illustrating the uncontrollability of NP and the difficulty in managing and coping with it.

Although participants used a wide range of metaphors to describe pain, Alice acknowledged such language may not always be adequate:

You can only explain so much of it, and there's a lot more to it than that, which I can't really explain.

Alice, inpatient: lines 176-177

Alice explains that describing NP is a task made difficult by the lack of experience of NP, and the lack of comparable feelings. People with SCI, therefore, may be able to describe their pain situation to some extent, but articulating completely sufficient accounts of its 
intensity and the distress induced, whilst obtaining understandings from those without SCI may not be completely possible.

\section{Discussion}

This study presents a novel, mixed-methods approach to the study of metaphor use when describing NP after SCI. The qualitative interpretations, therefore, are provided with further contextualization from the quantitative analyses. CA found that outpatients used a larger range of metaphors more frequently, than inpatients. Females used metaphors more often than males, whilst older participants were less likely to describe pain through metaphor. Scoping analyses indicated that those under two years since injury may be more likely to use metaphors to conceptualise NP than those over two years post-injury. Pain intensity was not associated with metaphor use. IPA revealed three themes with regard to metaphor use: (1) Pain as a Personal Attack, describing NP in terms of its uncontrollability, (2) The Desire to be Understood, which drew on comparisons that those without SCI may be able to relate to, and (3) Conveying Distress Without Adequate Terminology, in which the distress induced by NP was described with difficulty. Participants had not experienced NP prior to injury, and only had pre-SCI experiences to rely on as a means of describing NP. Consequently, it was acknowledged that even these descriptions were not enough.

Females in this study used more metaphors than males. Growing evidence suggests that communication styles differ between sexes in terms of pain (Hoffmann \& Tarzian, 2001; Keogh, 2006; Bernards, Keogh, \& Lima, 2008). Hoffmann and Tarzian (2001) suggest that females cope with pain in a social manner, seeking guidance from healthcare providers, whilst males are more likely to accept or ignore pain. Males are likely to use fewer words and less descriptive language when focusing on painful events, whereas women are more likely to focus on sensory aspects of pain (Strong, Mathews, Sussex, New, Hoey, \& Mitchell, 2009). 
This difference may be explained by gender role expectations; males are generally taught to be less expressive of pain, and more stoic, than females (Unruh, 1996), whilst females may be more willing to disclose pain (Robinson et al., 2001). Females in this study may have felt better able to discuss their pain, due to differences in conditioning and/or culture. Disclosing pain, however, is a complex social and psychological process, and further work examining gender differences in pain reporting is warranted.

Outpatients used a wider range of metaphors, and used them more often, than inpatients. Previous research has not addressed the role of patient status and metaphor use. It is possible that HCPs who may be more likely to understand the NP experience, are not as easily accessed by outpatients living in the community, compared with those in inpatient settings. Hearn, Cotter, Fine, and Finlay (2015) found that outpatients with SCI made attempts to isolate themselves from friends and family, in order to protect them from the impact of NP. This may make outpatients more likely to use metaphors to elicit empathy from others who may be less likely to understand the experience.

This study found that age was related to metaphor use. Younger participants were found to engage in metaphor use more often than older participants. This may be related to cause of injury, with half of those aged 50 and over sustaining their injury through a fall, two through RTAs, and two through non-traumatic means. None of those aged 49 and under had sustained their injury through a fall, but via RTAs, swimming and work-related incidents, and non-traumatic means. It may be argued that those who are younger sustained their injuries through means more distressing than a fall, subsequently causing more distress that may manifest itself in communication strategies. No literature is available to support this, however, and this area may require further research to clarify.

It may be argued that older adults used fewer metaphors as a result of age-related increased stoicism, which involves the regulation of emotions, increased self-reliance, and 
emotional 'toughness' (Wagstaff \& Rowledge, 1995). Amplified desires to avoid help-seeking behaviour (Helme \& Gibson, 2001; Yong, 2006) may be reflected in refusals to acknowledge pain and discuss its impact via metaphor. Further, the language that older individuals use may be representative of a perceptual shift towards positive information and emotion regulation (Carstensen, Pasupathi, Mayr, \& Nesselroade, 2000). Higher levels of stoicism, and reduced focus upon negative events like NP, may explain why older participants used fewer metaphors when describing their pain.

Time since injury may be implicated in language use, or desire to conceptualise NP after SCI. It may be that between one and two years post-injury acceptance and willingness to have pain may have increased, with concerns surrounding NP reduced. Risdon, Eccleston, Crombez, and McCracken (2003) suggest that pain acceptance occurs when patients acknowledge that a cure is unlikely, and when focus shifts from pain to other aspects of life. Further, the coping strategies adopted may change over time, depending on their suitability to the patient. Coping strategies have been associated with psychosocial adaptation to SCI (Livneh \& Martz, 2014), and change over time may be associated with the reduction in metaphor use. However, the small number of participants limits the extent to which conclusions can be drawn.

Metaphors used by participants in this study to describe pain were wide-ranging, emphasising the threat of pain. Some of these metaphors (burning, pins and needles, electric shock) are reflected by other groups, including HIV, diabetic, and post-stroke NP (Freeman, Baron, Bouhassira, Cabrera, \& Emir, 2014), suggesting that communication of NP may be an important priority, and that the following implications may be relevant to other groups with NP. Metaphors may have been used in attempts to provoke emotional responses, empathy, and understanding, from the listener (Semino, 2010). Semino argues that metaphor use can provide the basis for empathic responses, enriching understanding through its ability to elicit 
embodied simulation by the listener, in which experiences are understood in terms of previous experiences and memories. Referring to potential causes of bodily harm may enhance the listener's ability to relate to the experience, due to previous experience (Wilson \& Gibbs, 2007), knowledge (Matlock, Ramscar, \& Boroditsky, 2005), or prediction, in which introspection of one's cognitions and emotions allows conclusions to be drawn (Barsalou, 2008). Participants in this study may have opted to use metaphor in order to facilitate such perspective-taking, imaginative simulation, empathy, and understanding. Females may have adopted the use of more metaphors in order to obtain such understanding and empathy.

Whilst metaphors regarding pain may be motivated by positive intentions, such as obtaining empathic responses, they may induce and exacerbate distress by highlighting patients' perceptions of NP as inevitable and 'punishment', particularly when describing pain as 'purgatory' and 'torture'. The communal coping model of pain (Sullivan, 2012) states that catastrophising is a form of interpersonal communication used to cope with pain, motivated by proximity or support-seeking, and to solicit empathic responses. However, catastrophising is a significant predictor of pain behaviour (Thibault, Loisel, Durand, \& Sullivan, 2008) and it is suggested that metaphor use may be a form of catastrophising (Jamani and Clyde, 2008), causing the sufferer to pay increased attention to the pain. Such language use may increase the risk of negative emotional states and hypervigilance (Holmes, Arntz, \& Smucker, 2007; Villemure \& Bushnell, 2009). Therefore, using metaphors to attain social support increases focus placed upon threatening appraisals of pain, suggesting a need for communication skills training for both patients and HCPs in order to identify such language and reduce its negative impact. Future work may explore how catastrophising in those with NP affects the use of metaphors, and how the development of strong therapeutic alliances and metaphor-based techniques influence pain-related fear, anxiety, and disability beliefs. 


\section{Clinical Implications}

Re-analysing data from previous research found demographic differences in metaphor use by those with chronic NP following SCI. The use of metaphor for communicating NP enabled enhanced understanding of the lived experience to be reached. However, such metaphor use may also be indicative of psychological distress, or difficulty coping. Sensitivity to the use of metaphor during encounters with patients in chronic pain may improve the quality of healthcare provision and understanding of patients' experiences (Loftus, 2011), and consultants in one study agreed that using metaphors enhance communication between themselves and patients (Arroliga, Newman, Longworth, \& Stoller, 2002). Integrating the consideration of language use in consultations can allow for a deeper understanding and better identification of patients' current cognitive appraisals of NP, and could facilitate behaviour change.

Evaluating pain-related metaphor use could deliver benefits when used in adjunct to standard treatment (Villemure \& Bushnell, 2009). Those presenting with high levels of distress alongside frequent metaphor use may reap benefits from interventions such as rescripting (van der Kolk \& van der Hart, 1989). This involves identifying cognitions that maintain distress (Roth \& Fonagy, 1996), and attempts to transform thought content to less distressing images by promoting positive imagery (Holmes, Arntz, \& Smucker, 2007). Philips and Samson (2012) found that rescripting reduced negative appraisals of pain in a general chronic pain sample, and $49 \%$ of their participants reported no pain during the procedure.

Rescripting may, therefore, be viable for those with NP following SCI to promote positive appraisals and emotion regulation by identifying circumstances in which metaphor use maintains distress. However, it may be possible that rescripting metaphorical images of pain could remove a valuable communicative tool for obtaining empathy and facilitating 
understanding. Although more evidence is required, targeting image-based cognitions may be a viable intervention for those with NP after SCI. Further, the training of healthcare professionals in responding to, re-using, and exploring metaphors used by those in pain, may increase listener empathy, as well as the patient's coping.

\section{Limitations and Future Research}

The nature of IPA allows findings to be illuminative, rather than definitive (Smith, Flowers, \& Larkin, 2009). Metaphors used by others with NP, and how they are implemented and understood, may vary dependent on subjective context and experience. Future research should explore quantitative measures of metaphor use, and their association with pain-related catastrophising, depression, and anxiety, across chronic pain populations. It would be valuable to investigate patient-identified purposes of metaphor use, and the extent to which metaphors facilitate perspective-taking and empathy in social support. Such studies should involve larger samples, with the aim to move towards making more general, wider population-based claims.

The quantitative analyses presented in this work arise from a small, self-selecting sample, potentially representing a group of people more willing to discuss pain with a stranger than those who chose not to take part, and limits the extent to which the results can be generalised. Further, it is unknown as to whether those taking part in this study used metaphors as an unconscious reflex, or consciously (Charteris-Black 2004). A replication study with a focus on metaphor use is required to assess the extent to which the themes and statistical analyses are replicable, the consistency of metaphor use within this population, and the extent to which people choose to engage in metaphor use as a communication strategy, or whether this is an unconscious reflex in response to NP. This would offer evidence-based rationale for the use of interventions such as rescripting in the SCI population. Additional 
research should identify the psychological, social, and physiological mechanisms involved in metaphor use as a communication tool, in SCI and general chronic pain populations. The process of change that appears to occur between one and two years post-injury should be explored in order to enhance knowledge on how and why metaphor use changes over time. Improved comprehension of pain-related metaphor use may lead to the effective development and/or integration of treatment approaches and interventions for those living with chronic pain, in order to improve adaptive coping and reduce catastrophic thinking.

\section{Acknowledgements}

We would like to thank Dr. XXX, and all of the participants and staff at XXX, for their time and co-operation.

\section{Disclosure Statement}

The authors declare that there are no conflicts of interest, and agree to the publication of this article.

\section{References}

Arroliga, A. C., Newman, S., Longworth, D. L., Stoller, J. K. (2002). Metaphorical Medicine: Using Metaphors to Enhance Communication with Patients Who Have Pulmonary Disease. Annals of Internal Medicine, 137, 376-379. doi:10.7326/0003-4819-1375_Part_1-200209030-00037

Barsalou, L. W. (2008). Grounded cognition. Annual Review of Psychology, 59, 617-45. 
Becker, A. H. (1997). Emergent and common features influence metaphor interpretation. Metaphor and Symbol, 12, 243-259.

Bennett, M. (2001). The LANSS Pain Scale: the Leeds assessment of neuropathic symptoms and signs. Pain, 92(1-2), 147-157.

Berelson, B. (1952). Content analysis in communication research. Free Press.

Bernards, S. F., Keogh, E., Lima, M. L. (2008). Bridging the gap between pain and gender research: a selective literature review. European Journal of Pain, 12, 427-40.

Bourke, J. (2014). The Story of Pain. Oxford University Press: London.

Carstensen, L. L., Pasupathi, M., Mayr, U., \& Nesselroade, J. R. (2000). Emotional experience in everyday life across the adult life span. Journal of Personality and Social Psychology, 79, 614-655.

Charteris-Black, J. (2004). Corpus approaches to critical metaphor analysis. Basingstoke: Palgrave Macmillan.

Freeman, R., Baron, R., Bouhassira, D., Cabrera, J., \& Emir, B. (2014). Sensory profiles of patients with neuropathic pain based on the neuropathic pain symptoms and signs. Pain, 155, 367-376.

Hearn, J. H., Cotter, I., Fine, P., \& A. Finlay, K. (2015). Living with chronic neuropathic pain after spinal cord injury: an interpretative phenomenological analysis of community experience. Disability and Rehabilitation, 37(23), 2203-2211.

Helme, R. D., \& Gibson, S. J. (2001). The epidemiology of pain in elderly people. In: Ferrell, B. A. (Ed). Clinics in Geriatric Medicine. Vol. 17; pp. 417-431.

Hoffmann, D. E., \& Tarzian, A. J. (2001). The Girl Who Cried Pain: A Bias Against Women in the Treatment of Pain. Journal of Law, Medicine \& Ethics, 28, 13-27. 
Holmes, E. A., Arntz, A., \& Smucker, M. R. (2007). Imagery rescripting in cognitive behaviour therapy: Images, treatment techniques and outcomes. Journal of Behavior Therapy and Experimental Psychiatry, 38(4), 297-305.

Jamani, N., \& Clyde, Z. (2008). Treatment of pain-related fear in chronic (persistent) pain: the role of safety-seeking behaviour and imagery. The Cognitive Behaviour Therapist, $1(1), 3-15$.

Kennedy, P., Frankel, H., Gardner, B., \& Nuseibeh, I. (1997). Factors associated with acute and chronic pain following traumatic spinal cord injuries. Spinal Cord, 35(12), 814817.

Keogh, E. (2006). Sex and gender differences in pain: A selective review of biological and psychosocial factors. Journal of Men's Health \& Gender, 3(3), 236-243.

Livneh, H., \& Martz, E. (2014). Coping strategies and resources as predictors of psychosocial adaptation among people with spinal cord injury. Rehabilitation Psychology. 59(3), 329-339.

Loftus, S. (2011). Pain and its metaphors: A dialogical approach. Journal of Medical Humanities, 32(3), 213-230.

Lyddon, W. J., Clay, A. L., \& Sparks, C. L. (2001). Metaphor and Change in Counseling. Journal of Counseling \& Development, 79(3), 269-274.

Matlock, T., Ramscar, M., Boroditsky, L. (2005). On the experiential link between spatial and temporal language. Cognitive Science, 29(4), 655-64.

Melzack, R. (1975). The McGill Pain Questionnaire: major properties and scoring methods. Pain, 1(3), 277-99.

Modirian, E., Pirouzi, P., Soroush, M., Karbalaei-Esmaeili, S., Shojaei, H., \& Zamani, H. (2010). Chronic pain after spinal cord injury: results of a long-term study. Pain Medicine, 11(7), 1037-1043. 
Philips, H. C. (2011). Imagery and Pain: The Prevalence, Characteristics, and Potency of Imagery Associated with Pain. Behavioural and Cognitive Psychotherapy, 39(05), 523-540. http://doi.org/10.1017/S1352465811000282

Philips, H. C., \& Samson, D. (2012). The Rescripting of Pain Images. Behavioural and Cognitive Psychotherapy, 40(05), 558-576.

Radley, A., \& Chamberlain, K. (2001). Health psychology and the study of the case: from method to analytic concern. Social Science \& Medicine, 53(3), 321-332.

Risdon, A., Eccleston, C., Crombez, G., \& McCracken, L. (2003). How can we learn to live with pain? A $Q$-methoological analysis of the diverse understandings of acceptance and chronic pain. Social Science and Medicine. 56(2), 375-386.

Robinson, M. E., Riley, J. L., Myers, C. D., Paps, R. K., Wise, E. A., Waxenberg, L. B., \& Fillingim, R. B. (2001). Gender role expectations of pain: relationship to sex differences in pain. The Journal of Pain, 2, 251-7.

Roth, A., \& Fonagy, P. (1996). What works for whom? A critical review of psychotherapy research. New York: Guilford Press.

Semino, E. (2010). Descriptions of pain, metaphor, and embodied simulation. Metaphor \& Symbol, 25(4), 205-26.

Siddall, P. J., McClelland, J. M., Rutkowski, S. B., \& Cousins, M. J. (2003). A longitudinal study of the prevalence and characteristics of pain in the first 5 years following spinal cord injury. Pain, 103(3), 249-257.

Smith, J. A., Flowers, P., \& Larkin, M. (2009). Interpretive Phenomenological Analysis: Theory, Method and Research. London: SAGE Publications Ltd.

Smith, J.A. \& Osborn, M. (2003). Interpretative Phenomenological Analysis. In J.A. Smith (Ed.), Qualitative Psychology: A Practical Guide to Research Methods. London: Sage. 
Smith, B. M. \& Sparkes, A. C. (2004). Men, sport, and spinal cord injury: an analysis of metaphors and narrative types. Disability \& Society, 19(6), 613-626

Strong, J., Mathews, T., Sussex, R., New, F., Hoey, S., \& Mitchell, G. (2009). Pain language and gender differences when describing a past pain event. Pain, 5(1-2), 86-95.

Sullivan, M. J. L. (2012). The Communal Coping Model of Pain Catastrophising: Clinical and Research Implications. Canadian Psychology, 53(1), 32-41.

Thibault, P., Loisel, P., Durand, M. J., \& Sullivan, M. J. L. (2008). Psychological predictors of pain expression and activity intolerance in chronic pain patients. Pain, 139, 47-54.

Unruh, A. M. (1996). Review article - gender variations in clinical pain experience. Pain, 65, $123-67$.

Van der Kolk, B. A., \& van der Hart, O. (1989). Pierre Janet and the breakdown of adaptation in psychological trauma. The American Journal of Psychiatry, 146(12), 1530-1540.

Villemure, C., \& Bushnell, M. C. (2009). Mood Influences supraspinal pain processing separately from attention. The Journal of Neuroscience, 29(3), 705-15.

Wagstaff, G. F., Rowledge, A. M. (1995) Stoicism: its relation to gender, attitudes toward poverty, and reactions to emotive material. Journal of Social Psychology, 135, 181184.

Werhagen L, Budh CN, Hultling C, Molander C. (2004). Neuropathic pain after traumatic spinal cord injury--relations to gender, spinal level, completeness, and age at the time of injury. Spinal Cord 42(12):665-73.

Wilson, N. L., \& Gibbs, R. W. (2007) Real and imagined body movement primes metaphor comprehension. Cognitive Science, 31(4), 721-31.

Yong, H.-H. (2006). Can attitudes of stoicism and cautiousness explain observed age-related variation in levels of self-rated pain, mood disturbance and functional interference in 
chronic pain patients? European Journal of Pain, 10(5), 399-407. http://doi.org/10.1016/j.ejpain.2005.05.004 